Queen's University Belfast Cite this as: BMJ2022;376:0131 http://dx.doi.org/10.1136/bmj.0131 Published: 18 January 2022

\section{Online medical school-a win for accessibility?}

The changes medical schools made during the pandemic could pave the way for an education system that is more responsive to the needs of people with disabilities, writes Patrick Doherty

\section{Patrick Doherty second year medical student}

Since the onset of the covid-19 pandemic, medical school curricula have proven to be much more adaptable than we may have thought. Every UK medical school has had to move from traditional teaching in lecture theatres and seminar rooms to finding socially distanced solutions through online education tools. As a disabled medical student who lives with epilepsy, ulcerative colitis, and reactive arthritis, I wonder if the proof of concept of "online medical schools" could finally be a step in the right direction to improve accessibility for disabled people.

The GMC's “Welcomed and valued” guidance rightly recognises that diversity among medical professionals is essential to reflect the patient population, many of whom are disabled. ${ }^{1}$ It is unfortunate then that many disabled people still do not feel able to fully disclose their disabilities in the medical profession. This reality is demonstrated by the large disparity between the proportion of NHS staff who identify as disabled in anonymous surveys versus on the NHS electronic staff record: $19 \%$ and $3 \%$, respectively. ${ }^{2}$ The problems faced by disabled, chronically ill, or neurodiverse people are not homogeneous. Yet the adaptations made to the medical curricula during the pandemic may well provide a framework for personalising teaching to the needs of this group, who so often struggle with a medical culture that is not inclusive of disabled people and in which the voices of disabled leaders are not always heard.

\section{Flexibility is key}

The BMA found that the most common adjustment requested by disabled doctors and medical students to make medicine more accessible was flexibility. ${ }^{2}$ As a medical student with disabilities, I'm hardly surprised that this is a frequent obstacle in a course renowned for the intensity of its contact hours. Yet flexibility has been forced upon many medical schools over the course of the pandemic, changing how and when they teach students. Disabled students like me have seen the realisation of new teaching methods that would not have been conceivably rolled out before the pandemic, but which have dramatically improved our learning experience. Adaptive education formats have included cadaver laboratories being replaced by using 3D app technology, recorded lectures, seminars being delivered via video link, and clinical teaching through telemedicine calls.

One survey of students across 39 medical schools found that flexible learning was perceived as the most useful benefit of online teaching. ${ }^{3}$ For medical students who are disabled, this flexibility means more than personal convenience and should be viewed through multiple frames.
For starters, online learning may help disabled students who would otherwise struggle with transport to attend teaching sessions (if they are unable to drive or find public transport inaccessible). By not having to move between classes physically, students can decide in what environment they would like to learn while limiting the fatigue created by having to get around campuses. Considering the fluctuating nature of living with a chronic condition, the constraint of having to follow a fixed timetable is often significant and the ability to study material asynchronously is beneficial. Finally, there are some students whose learning needs mean they require additional time or would benefit from recorded teaching, which allows them to rewind or pause material to maximise their learning.

\section{New ways of communicating}

All students are likely to benefit from these changes though, which prepare us for future clinical practice, particularly considering the rise of remote consultations. Students' adaptation of their communication skills to work remotely with patients and colleagues alike may well create a generation of doctors who are more adept at communicating with patients in new ways. This has the potential to improve accessibility for patients and allow the NHS to be led more by staff and service users.

Online learning is not without its problems of course: the survey I previously referenced noted that students had an overall higher preference for face-to-face teaching for reasons such as lack of interaction with their peers and poor access to the internet. ${ }^{3}$

Universities could respond to these challenges by lobbying for improved connectivity and by creating learning resources with more interactivity, improved support structures, and more collaborative teaching. These problems are far from insurmountable for a profession that has shown itself capable of adapting dynamically to a pandemic.

\section{Breaking down barriers}

While I have had a very proactive disability support team at my medical school, this is not the predominant experience. With only $41 \%$ of disabled doctors and medical students feeling that they have had tangible benefits from disclosing their disability at work or medical school, ${ }^{2}$ it is clear that there is a great deal of work to be done in meeting the needs of those with a disability. As online learning looks like it's here to stay in some form, and with most schools still using hybrid teaching, disabled students ought to be considered and consulted on how these short term changes could become more long term solutions. I'd argue that the adaptations we've seen 
to medical education delivery could form the basis of a long term plan for more flexible teaching, which is reflected in and protected by official policies.

Disabled medical students and healthcare professionals have a unique perspective to offer due to their lived experiences. If medical education and the healthcare system is to be more accommodating of disabled people, we must now try to learn from the adaptations to learning made throughout the pandemic. Universities, hospitals, and GP surgeries can build on what has gone well and continue to break down the barriers that historically have made medical education and practice inaccessible to disabled people.

It is my hope that we can begin now to implement the flexibility that disabled doctors and students have long craved. ${ }^{4}$ To do this we can begin by capitalising on the digital conferencing solutions that can facilitate remote teaching and, where appropriate, remote simulated patient interactions and, as a profession, be more willing to accommodate different methods and hours of learning. This, I believe, may go some way to creating a modern medical education system that recognises the immense contribution and value of disabled people.

Competing interests: none declared.

Provenance and peer review: not commissioned, not peer reviewed.

1 Welcomed and valued. General Medical Council. https://www.gmc-uk.org/education/standardsguidance-and-curricula/guidance/welcomed-and-valued

2 Disability in the medical profession: Survey findings 2020. The BMA. 2020. https://www.bma.org.uk/media/2923/bma-disability-in-the-medical-profession.pdf

3 Dost S, Hossain A, Shehab M, Abdelwahed A, Al-Nusair L. Perceptions of medical students towards online teaching during the COVID-19 pandemic: a national cross-sectional survey of 2721 UK medical students. BMJ Open 2020;10:e042378. doi: 10.1136/bmjopen-2020-042378. pmid: 33154063

4 BMA lobbying: Disability in the medical profession. The BMA. 6 January 2022. https://www.bma.org.uk/advice-and-support/nhs-delivery-and-workforce/workforce/disabilityin-the-medical-profession 